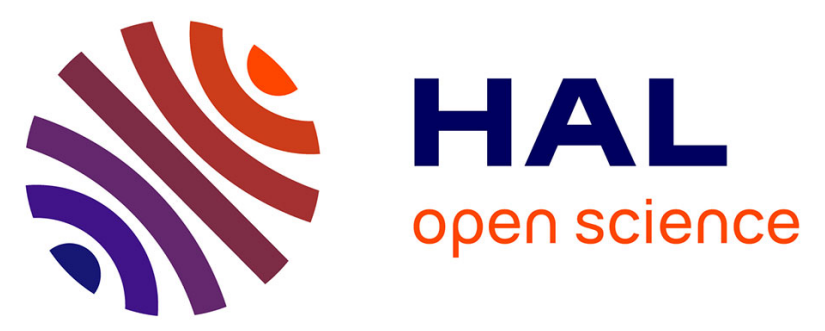

\title{
Strain-based parameters for infarct localization: evaluation via a learning algorithm on a synthetic database of pathological hearts
}

Gerardo Rumindo, Nicolas Duchateau, Pierre Croisille, Jacques Ohayon, Patrick Clarysse

\section{To cite this version:}

Gerardo Rumindo, Nicolas Duchateau, Pierre Croisille, Jacques Ohayon, Patrick Clarysse. Strainbased parameters for infarct localization: evaluation via a learning algorithm on a synthetic database of pathological hearts. 9th International Conference, Functional Imaging and Modeling of the Heart (FIMH), 2017, Toronto, Canada. pp.106-114. hal-01829278

\section{HAL Id: hal-01829278 \\ https://hal.science/hal-01829278}

Submitted on 3 Jul 2018

HAL is a multi-disciplinary open access archive for the deposit and dissemination of scientific research documents, whether they are published or not. The documents may come from teaching and research institutions in France or abroad, or from public or private research centers.
L'archive ouverte pluridisciplinaire HAL, est destinée au dépôt et à la diffusion de documents scientifiques de niveau recherche, publiés ou non, émanant des établissements d'enseignement et de recherche français ou étrangers, des laboratoires publics ou privés. 


\title{
Strain-Based Parameters for Infarct Localization: Evaluation via a Learning Algorithm on a Synthetic Database of Pathological Hearts
}

\author{
GK Rumindo ${ }^{1 *}, \mathrm{~N}_{\text {Duchateau }}{ }^{1}, \mathrm{P}$ Croisille $^{1}, \mathrm{~J}$ Ohayon $^{2}$, and P Clarysse ${ }^{1}$ \\ 1 Univ.Lyon, INSA-Lyon, Université Claude Bernard Lyon 1, UJM-Saint Etienne, \\ CNRS, Inserm, CREATIS UMR 5220, U1206, LYON, France \\ Kenny . Rumindo@creatis.insa-lyon.fr \\ ${ }^{2}$ University of Savoie Mont Blanc, Polytech Annecy-Chambéry, Laboratory \\ TIMC-IMAG/DyCTiM2, UGA, CNRS, Grenoble, France
}

\begin{abstract}
Localization of infarcted regions is essential to determine the most appropriate treatment for patients with cardiac ischemia. Myocardial strain partially reflects the location of infarcted regions, which demonstrated potential use in clinical practice. However, strain patterns are complex and simple thresholding is not sufficient to locate the infarcts. Besides, many strain-based parameters exist and their sensitivities to myocardial infarcts have not been directly investigated. In our study, we propose to evaluate nine strain-based parameters to locate infarcted regions. For this purpose, we designed a large database $(n=200)$ of synthetic pathological finite-element heart models from 5 real healthy left ventricle geometries. The infarcts were incorporated with random location, shape and degree of severity. In addition, we used a state-of-theart learning algorithm to link deformation patterns and infarct location. Based on our evaluation, we propose to sort the strain-based parameters into three groups according to their performances in locating infarcts.
\end{abstract}

Keywords: Finite-element model, myocardial infarct, myocardial strain, infarct diagnosis, machine learning

\section{Introduction}

The clinical value of determining myocardial viability to help the physicians decide on the best treatment for patients with cardiac ischemia has been established [5]. Late Gadolinium Enhancement - an MR imaging method - is generally accepted as the gold standard to assess myocardial viability and therefore to locate myocardial infarcts. However, it requires contrast injection, is costly and not available for all patients. Myocardial strains - extracted from cardiac MR [14] or echocardiography [3] - have also been used to identify dysfunctional regions of the heart [1]. However, due to the complex strain patterns, simple thresholding is not sufficient and further processing is required [12]. Learning-based algorithms 
have been investigated for better detecting [9] and locating [2,4] infarcts from strain data. These methods are promising and can be generalized to different modalities and subjects, in addition to showing high accuracy of infarct detection.

Several strain-based parameters have been investigated for infarct localization. However, many more exist and their potential has not been evaluated. In our study, we propose to evaluate the following nine strain-based parameters; principal strain, effective strain, fractional anisotropy [13], three local directional strains (radial, circumferential, longitudinal), and three stretch-dependent invariants (in fiber, cross-fiber, and sheet-normal directions). They were assessed in terms of their localization performance (sensitivity and specificity) given a learning algorithm to locate the infarcts. The algorithm uses a regression to find the transfer function between the tested parameters and the infarct location [4]. To do so, we designed a large database of synthetically-generated pathological cases that incorporates infarcts with different locations, shapes, sizes, and degrees of severity. Notably, we demonstrated that by properly designing and exploiting a large and sufficiently varied database of pathological cases, we were able to really push the limits of each strain parameter used in the detection algorithm and to better estimate their localization performances.

\section{Materials and methods}

Five LV meshes (3552 hexahedral elements) from healthy volunteers were obtained from an open access source [6]. A finite-element model of each healthy LV in the diastolic (filling) phase was simulated. Afterwards, 40 pathological cases were generated based on each LV geometry - resulting in 200 pathological cases - and the nine strain-based parameters were computed. Finally, the learning algorithm was applied to evaluate the localization performance of each parameter.

\subsection{Database of pathological cases}

Simulation of healthy cases. The diastolic simulation for the healthy LV model follows the principles detailed in $[6,11]$. Rule-based fiber orientation was incorporated into the LV geometry by first defining a pseudoprolate spheroidal coordinate system that consists of radial (orthogonal to the LV surface), circumferential and longitudinal (tangential to the LV surface) directions. Subsequently, an orthogonal fiber coordinate system was defined, which is comprised of fiber, sheet and sheet-normal directions. The fibers are oriented with an elevation angle distribution of $-60^{\circ}$ to $+60^{\circ}$ from the epicardium to the endocardium [7].

The constitutive law was the transversely isotropic Fung-type law [6] (Eq. 1). The strain energy density function is divided into two parts; the isochoric term, which is based on the Green-Lagrange strain tensor $\mathbf{E}$, and the volumetric term: 


$$
\begin{gathered}
\mathbf{W}=\overbrace{\frac{C}{2}\left(e^{Q}-1\right)}^{\text {Isochoric term }}+\overbrace{\frac{1}{D}\left(\frac{J^{2}-1}{2}-\ln J\right)}^{\text {Volumetric term }} \\
\underbrace{Q=b_{f} E_{f f}^{2}+b_{t} E_{s s}^{2}+b_{t} E_{n n}^{2}+b_{t}\left(E_{s n}^{2}+E_{n s}^{2}\right)+b_{f s}\left(E_{f s}^{2}+E_{s f}^{2}+E_{f n}^{2}+E_{n f}^{2}\right)}_{\text {Exponential terms of the isochoric part; } \boldsymbol{E}: \text { Green-Lagrange strain tensor }}
\end{gathered}
$$

where $C, b_{f}, b_{t}$ and $b_{f s}$ are the material parameters to be personalized for each subject; $J$ is the determinant of the deformation gradient tensor $\mathbf{F}$; whereas $D$ was set to 0.001 to enforce quasi-incompressibility. The subscripts $f, s$ and $n$ denote the fiber, sheet and sheet-normal directions, respectively. Active tension was not taken into account as we only simulated the diastolic phase in this study. The models were solved using the finite-element software ABAQUS ${ }^{1}$, and the constitutive law was implemented in ABAQUS user material subroutine UMAT.

The material parameters were personalized for each subject based on the end-diastolic pressure-volume relationships described in [8]. The beginning- and end-diastolic volumes of each subject were known; the corresponding pressures were set to 0 and $9 \mathrm{mmHg}$, respectively.

Simulation of pathological cases. Forty synthetically-generated cases were generated from each of the 5 healthy LV models, resulting in 200 cases. Infarct regions were incorporated through a binary value at each mesh element. These regions were defined as follows: first, the intra-ventricular junction and apex of each LV were manually selected from the MR images, which enabled us to define the left anterior descending (LAD) coronary artery territory on each LV. The infarcts had truncated ellipsoidal or spheroidal shapes with arbitrary sizes ranging from 0.5-99.7 ml, whose center points were constrained to be on the endocardial surface and within the LAD territory. This territory was chosen due to its high prevalence [10]. The material properties of the infarct were set to be stiffer compared to the healthy ones, and they were assigned in a uniformlydistributed manner by changing the material parameter $C$ (Eq. 1) from 1 up to 2.50 times of the personalized healthy values. The resulting infarcts have an average myocardial mass of $22.9 \pm 21.3 \mathrm{~g}$ and an average volume of $21.6 \pm 20.1$ $\mathrm{ml}$, corresponding to $15.6 \pm 13.2 \%$ of the myocardium total volume.

\subsection{Strain-based parameters}

Nine strain-based parameters were evaluated in this study (Table 1). Starting from the deformation gradient tensor $\mathbf{F}$ extracted from ABAQUS simulations, the right Cauchy stretch tensor $\mathbf{C}$ and the Green-Lagrange strain tensor $\mathbf{E}$ were calculated: $\mathbf{C}=\mathbf{F}^{T} . \mathbf{F} ; \mathbf{E}=\frac{1}{2}(\mathbf{C}-\boldsymbol{I})$; where $\boldsymbol{I}$ is the identity tensor.

\footnotetext{
${ }^{1}$ http://www.3ds.com/products-services/simulia/products/abaqus/
} 
Table 1: Strain-based parameters evaluated in this study

\begin{tabular}{|c|c|}
\hline Parameters & Formulae / Comments \\
\hline $\begin{array}{l}\text { Radial strain } \\
\text { Circ. strain } \\
\text { Long. strain }\end{array}$ & With respect to pseudoprolate coordinate system \\
\hline $\begin{array}{l}\text { Fiber invariant } \\
\text { Cross-fiber invariant } \\
\text { Sheet-normal invariant }\end{array}$ & $\begin{array}{l}\mathbf{f}_{0} \cdot\left(\mathbf{C f}_{0}\right) \quad \mathbf{f}_{0}: \text { fiber direction } \\
\mathbf{s}_{0} \cdot\left(\mathbf{C s}_{0}\right) \quad \mathbf{s}_{0}: \text { cross-fiber direction } \\
\mathbf{n}_{0} \cdot\left(\mathbf{C n}_{0}\right) \quad \mathbf{n}_{0}: \text { sheet-normal direction } \\
\end{array}$ \\
\hline Principal strain & The max positive Eigenvalue of $\mathbf{E}$ \\
\hline Effective strain & $\sqrt{\begin{array}{c}\frac{2}{9}\left[\left(E_{x x}-E_{y y}\right)^{2}+\left(E_{y y}-E_{z z}\right)^{2}+\left(E_{x x}-E_{z z}\right)^{2}\right. \\
\left.+6\left(E_{x y}^{2}+E_{y z}^{2}+E_{x z}^{2}\right)\right]\end{array}}$ \\
\hline Fractional anisotropy & $\begin{array}{ll}\sqrt{\frac{3}{2} \frac{\left(\lambda_{1}-\lambda\right)^{2}+\left(\lambda_{2}-\lambda\right)^{2}+\left(\lambda_{3}-\lambda\right)^{2}}{\lambda_{1}^{2}+\lambda_{2}^{2}+\lambda_{3}^{2}}} & \lambda=\left(\lambda_{1}+\lambda_{2}+\lambda_{3}\right) / 3 \\
& \lambda_{1}, \lambda_{2}, \lambda_{3} \text { : Eigenvalues of } \mathbf{E}\end{array}$ \\
\hline
\end{tabular}

\subsection{Infarct localization}

Data alignment. Due to the use of different geometries, the strain parameters were not directly comparable and spatial correspondence should be obtained. Thus, normalized local coordinates were computed on each geometry corresponding to the pseudoprolate coordinate system explained in Sec. 2.1. Then, a reference geometry was computed by Procrustes analysis with similarity transformation. Local coordinates were also computed for this reference geometry. Finally, local data (the strain parameters and the infarct binary labels) were transported to the reference geometry using the correspondence of the local coordinates. The data transportation involved interpolation, addressed by ridge
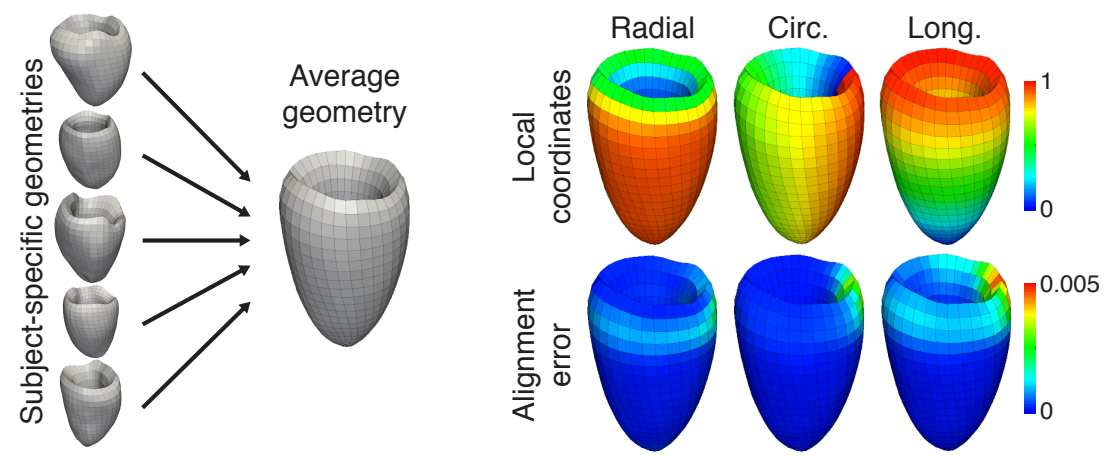

Figure 1: Left: The five LV and the calculated reference. Right: The local normalized pseudoprolate coordinates (radial, circumferential, longitudinal) and the average alignment error due to the interpolation of the local coordinates onto the reference. 


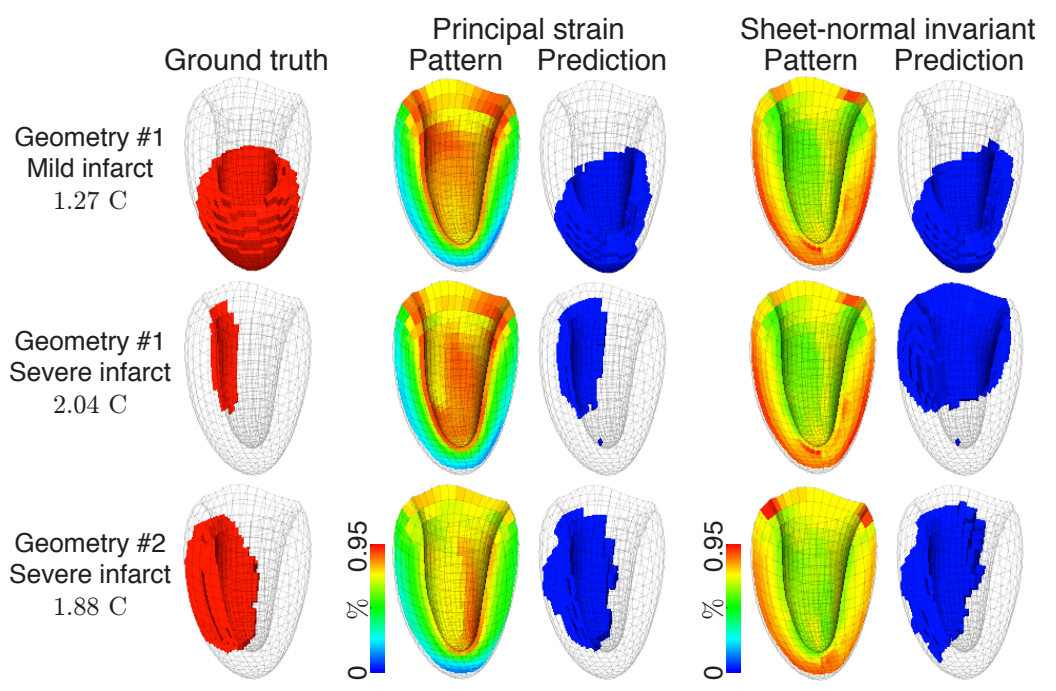

Figure 2: Infarct localization for some pathological cases based on principal strain and sheet-normal invariant; amongst the best and the worst parameters, respectively.

regression with a Gaussian kernel, with a balanced weight between similarity and regularization terms. The interpolation resulted in negligible errors. The whole process is summarized in Fig.1.

Regression. The aligned strain parameters and infarct location were treated as column vectors of length equal to the number of elements for the reference mesh. The link between each strain parameters and infarct location was learned via kernel ridge regression, inspired by the algorithm described in [4]. Direct regression was preferred over going through an intermediate space of reduced dimensionality, whose purpose is mainly for uncertainty modeling without substantially affecting the localization performance.

Each healthy LV geometry was used to generate 40 out of the total 200 pathological cases, which in turn might present some biases in the detection algorithm due to a substantial amount of relatively similar cases, thereby limiting the difference in deformation patterns and in the distribution of the evaluated parameters. To avoid this bias when a case was investigated, all other cases derived from the same geometry were not included in the training set. The training set was randomly constructed from the remaining cases with a certain percentage, which was in the range of $5-40 \%$ of the total population. The localization performance of each parameter was then tested against the size of the training set. The limit of $40 \%$ was set since it was observed that the algorithm was able to accurately detect the infarcts with this percentage of population as the training set.

The regression output consisted of a non-binary scalar value at each mesh element due to the linear combination of infarct locations. A binary localization 

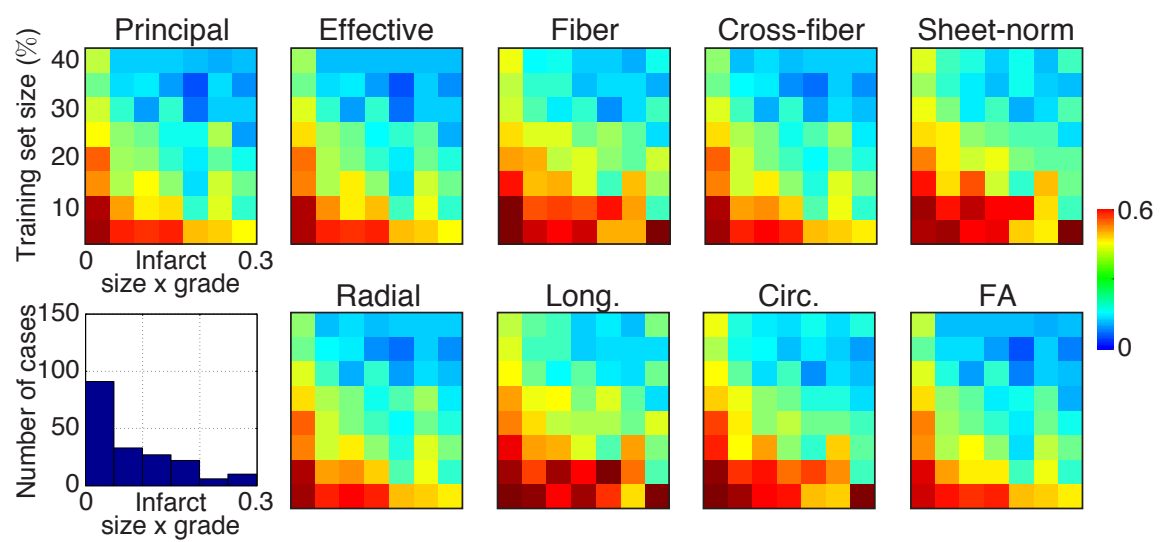

FA

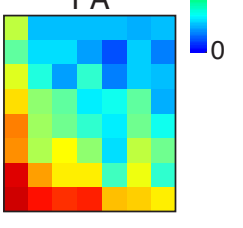

Figure 3: Performance of the strain-based parameters (median over the tested cases). The color bar depicts the distance to an ideal localization. Horizontal axis: a normalized combination of the degree of severity and the size of the infarct. Vertical axis: the percentage of the population used as the training set. The histogram shows the number of cases that correspond to the infarct size-severity measure on the horizontal axis.

of the infarct was obtained after thresholding this output by an appropriate value, as inspired by the two-step process described in [4]: 1) ROC analysis was first performed for each case to obtain a set of best individual thresholds. However, this analysis relies on the ground truth infarct location for each case, which is unrealistic in real-world clinical application, thus 2) the same optimal threshold was applied to all cases, whose values were chosen as the median of the set of the best individual thresholds. Thus, we ended up with a single consensual threshold, which is used in the rest of this paper.

\section{Results}

Fig. 2 depicts the infarct localization for pathological cases based on principal strain and sheet-normal invariant, which were observed as one of the best and worst parameters, respectively. The degree of severity of the infarct was based on how much stiffer it was in comparison to the healthy ones, with respect to the value of the material parameter $C$. The principal strain is able to localize the infarcts substantially better than the sheet-normal invariant. The infarct localization performance was evaluated in terms of sensitivity and specificity after thresholding the regression output by the consensual value as explained in Sec. 2.3. The results were summarized in terms of the distance to the ideal localization performance, namely a sensitivity and a specificity equal to 1 . Fig. 3 summarizes the performance of the nine strain-based parameters, with the color bar depicting the distance to an ideal localization. The horizontal axis represents a combination of the degree of severity and the size of the infarct normalized from 0 to 1 , where 0 signifies smaller infarcts and/or infarcts with closer material properties to the healthy ones; whereas the vertical axis represents 
the percentage of the population included in the training set. Lower values on the color bar signify better accuracy. In addition, the histogram presents the distribution of the number of pathological cases that correspond to each infarct size-severity bin on the horizontal axis. As expected, the localization performance of all parameters increases with bigger and more severe infarcts, as well as with the size of the training set. Based on this, the performance of the evaluated parameters can be divided into three groups. The best group consists of principal strain, effective strain, and fractional anisotropy. The second-best group consists of cross-fiber invariant and radial strain; whereas the last group consists of fiber and sheet-normal invariant, circumferential and longitudinal strain.

\section{Discussions}

Our study details a novel strategy for evaluating different strain-based parameters for infarct diagnosis. We combined finite-element modeling to generate a large synthetic database of pathological hearts based on healthy volunteers' data and a state-of-the-art learning algorithm to localize myocardial infarct. Nine strain-based parameters, several of which have yet to be investigated for infarct localization, were evaluated. The large database allowed us to push the algorithm to its limit and thoroughly evaluate the localization performance of each parameter.

The differences between the evaluated parameters were subtle, but we could identify them into three groups. The best group includes the principal strain, effective strain, and fractional anisotropy. Their performances were very similar. The second group shows slightly inferior, yet still good performance. It notably comprises the radial strain, which is readily available in clinical setting. It is interesting that the best parameters are those that are direction-independent, which was somewhat expected as they may be able to better highlight and extract the general distribution of myocardial deformation. However, the invariant in the fiber direction performed worse than the radial strain and the invariant in cross-fiber direction. The reason for this might be due to the boundary conditions applied for the diastolic-filling simulation, which forced the elements to deform radially as the volume of the cavity increases and the incompressibility of the element is enforced. This should be investigated further. In addition, the combination between parameters were not investigated and are also left for future work.

Our synthetic database was derived only from five LV geometries. Although these geometries come from MRI examinations of healthy volunteers - thereby ensuring that the personalized healthy material parameters are physiological - it is of interest in the future to be able to generate a database with larger variability in terms of geometries and subsequently deformation patterns. It is also well known that geometrical alterations of the LV might occur in chronic ischemic patients, i.e. the thickening and thinning of ventricular wall around the remote and infarcted myocardium, respectively. Since our database was derived from healthy volunteers, this effect was not taken into account. 
It is certainly essential to evaluate the infarct detection performance of the strain-based parameters on real clinical data of ischemic patients. However, the complexity in acquiring and processing strain data from images with clinical routine quality is still very challenging. Thus, investigation on clinical data is reserved for future studies. Additionally, the exclusion of clinical data allowed us to evaluate the strain-based parameters on a fully-controlled setting. Our study was also limited by the regression approach used for the learning, which relies on a global distance between deformation patterns and linearly combines the infarct candidates. Although the learning algorithm was simply used in our study as a state-of-the-art infarct detection tool to test the performance of the strain-based parameters, further work using different regression approaches or learning algorithms needs to be explored.

\section{Conclusions}

We have proposed a thorough evaluation of various strain-based parameters in locating myocardial infarct. We took advantage of finite-element modeling to generate a large database of pathological hearts and test the limits of a given localization algorithm against a variety of infarct configurations. Although the strain-based parameters only showed slightly different performance towards locating infarcts, we were able to divide them into three groups, which we showed to be coherent with physiological interpretations.

Acknowledgement. GK Rumindo is supported by the European Commission H2020 Marie Sklodowska-Curie European Training Network VPH-CaSE (www.vph-case.eu), grant agreement No 642612. This work was performed within the LABEX PRIMES (ANR-11-LABX-0063) of Université de Lyon, within the program "Investissements d'Avenir" (ANR-11-IDEX-0007) operated by the French National Research Agency (ANR); and the IMPULSION project from the Programme Avenir Lyon - St. Etienne.

\section{References}

1. Antoni ML, Mollema SA, Delgado V et al (2010) Prognostic importance of strain and strain rate after acute myocardial infarction. Eur Heart J 31: 1640-1647

2. Bleton H, Margeta J, Lombaert H, Delingette H, Ayache N. Myocardial infarct localization using neighborhood approximation forests. STACOM 2015, Munich, Germany

3. Dandel M, Lehmkuhl H, Knosalla C et al (2009) Strain and strain rate imaging by echocardiography - basic concepts and clinical applicability. Curr Cardiol Rev 5(2): 133-148

4. Duchateau N, De Craene M, Allain P et al (2016) Infarct localization from myocardial deformation: prediction and uncertainty quantification by regression from a low-dimensional space. IEEE Trans Med Imaging 35(10): 2340-2353

5. Flachskampf FA, Schmid M, Rost C et al (2011) Cardiac imaging after myocardial infarction. Eur Heart J 32(3): 272-23 
6. Genet M, Lee LC, Nguyen R et al (2014) Distribution of normal human left ventricular myofiber stress at end diastole and end systole: a target for in silico design of heart failure treatments. J Appl Physiol 117: 142-152

7. Holzapfel GA, Ogden RW (2009) Constitutive modelling of passive myocardium: a structurally-based framework for material characterization. Phil Trans R Soc A 367: $3445-3475$

8. Klotz S, Hay H, Dickstein ML et al (2006) Single-beat estimation of end-diastolic pressure-volume relationship: a novel method with potential for noninvasive application. Am J Physiol Heart Circ Physiol 291: H403-H412

9. Medrano-Garcia P, Zhang X, Suinesiaputra AA et al (2015) Statistical shape modelling of the left ventricle: myocardial infarct classification challenge. MICCAI STACOM 2015

10. Ortiz-Pérez JT, Rodriguez J, Meyers SN et al (2008) Correspondence between the 17 -segment model and coronary arteryal anatomy using contrast-enhanced cardiac magnetic resonance imaging. JACC Cardiovasc Imaging 1(3): 282-93

11. Rumindo GK, Ohayon J, Viallon M et al (2016) Comparison of different strainbased parameters to identify human left ventricular myocardial infarct during diastole: a 3D finite element study. CMBBE 2016, Tel Aviv, Israel

12. Sjøli B, Ørn S, Grenne B et al (2009) Diagnostic capability and reproducibility of strain by Doppler and by speckle tracking in patients with acute myocardial infarction. JACC Caridovasc Imaging 2: 24-33

13. Soleimanifard S, Abd-Elmoniem KZ, Agarwal HK et al (2010) Identification of myocardial infarction using three-dimensional strain tensor fractional anisotropy. Proc IEEE Int Symp Biomed Imaging 468-471

14. Wang H, Amini AA (2012) Cardiac motion and deformation recovery from MRI: a review. IEEE Trans Med Imaging 31(2): 487-503 